\title{
AVALIAÇÃO DE DIFERENTES MÉTODOS E ESTIMATIVA DE PARÂMETROS CINÉTICOS NA DEGRADAÇÃO TÉRMICA DE ANTOCIANINAS EM POLPA DE AMORA
}

\author{
J. M. S. CRISPIM ${ }^{1}$, N. L. SILVA ${ }^{1}$ e R. P. VIEIRA ${ }^{1}$ \\ ${ }^{1}$ Instituto Federal do Sul de Minas Gerais, Campus Pouso Alegre, Engenharia Química \\ E-mail para contato: ronierik.vieira@ifsuldeminas.edu.br
}

RESUMO - Este trabalho apresenta diferentes métodos de estimativa de parâmetros cinéticos na degradação térmica de antocianinas presente na polpa de Amora. O objetivo deste trabalho é avaliar os métodos de integral, diferencial e não linear e estimar os parâmetros cinéticos da equação em um reator batelada a volume constante com a concentração de antocianinas medida em função do tempo e em quatro temperaturas diferentes. $\mathrm{O}$ modelo cinético de ordem 1 apresentou o melhor ajuste, com os parâmetros da lei de Arrhenius, fator pré-exponencial $\left(k_{0}=1,06 \times 10^{6} \mathrm{~min}^{-1}\right)$ e energia de ativação $\left(E a=56,025 \mathrm{~kJ} \mathrm{~mol}^{-1}\right)$. A obtenção do modelo e seus parâmetros viabiliza a análise do processo de degradação térmica de antocianina em outras temperaturas.

\section{INTRODUÇÃO}

O Brasil é um dos principais produtores de frutas tropicais e o consumo delas em forma de polpas está em expansão no mercado consumidor devido estas apresentarem nutrientes e compostos essenciais à saúde humana (Kuskoski et al. 2006).

Um dos compostos essenciais são as antocianinas, pigmentos que vão desde a cor azulada a avermelhada (Alkema e Seager, 1982) sendo responsáveis pela coloração de frutas, como por exemplo, acerola, açaí, amora entre outras, e também pela coloração de vegetais e flores.

As antocianinas possuem diversas atividades biológicas e pesquisas tem mostrado que o consumo desse composto pelos seres humanos tem trazido muitos benefícios à saúde devido ao seu efeito preventivo contra várias doenças, podemos citar a sua atividade antioxidante que combate os radicais livres, atividade hepatoprotetor que age contra a diabetes, atividade antiinflamatória que previne doenças cardiovasculares, e atividade anticancerígena (Kumar e Pandey, 2013). Por isto, é importante que as polpas comercializadas apresentem elevadas quantidades desse composto.

Entretanto para a comercialização de polpas, elas precisam passar por um tratamento térmico, a pasteurização, na qual são empregadas temperaturas inferiores a $100^{\circ} \mathrm{C}$ com intuito de inibir o crescimento de microrganismos, aumentando o tempo de vida de prateleira do 
produto. Contudo as antocianinas são sensíveis, degradando-se na presença de oxigênio, a exposição à luz, a temperaturas superiores a ambiente, entre outros fatores (Lopes et al. 2007).

Portanto, o objetivo deste trabalho é avaliar os métodos de integral, diferencial e não linear para determinar o melhor modelo cinético. Além disso, objetiva-se, também, estimar os parâmetros cinéticos da equação de taxa, considerando um reator batelada a volume constante com a concentração de antocianinas medida em função do tempo e em quatro temperaturas diferentes. Como principal resultado deste trabalho, vislumbra-se obter um modelo cinético com validade experimental, viabilizando posteriores análises de perdas de antocianinas durante o processo de pasteurização da polpa de amora.

\section{MATERIAL E MÉTODOS}

Os dados experimentais de quantificação de antocianinas presentes na Polpa de Amora, em função do tempo e em quatro temperaturas diferentes $\left(60^{\circ} \mathrm{C}, 70^{\circ} \mathrm{C}, 80^{\circ} \mathrm{C}\right.$ e $\left.90^{\circ} \mathrm{C}\right)$ foram determinadas pelo do $\mathrm{pH}$ diferencial (Lee et al., 2005) utilizando um equipamento de UV-Vis para análise.

A partir dos dados experimentais, foram avaliados três métodos, sendo eles o método integral, diferencial e método não linear. Foi utilizado o software Excel para resolução dos cálculos considerando uma reação isotérmica em um reator batelada a volume constante.

\subsection{Método Integral}

Integrou-se a equação de taxa, Equação 1, na qual foram testados os modelos de ordem 0,1 e 2 para determinar qual seria o melhor modelo que representa a taxa de degradação térmica de antocianinas em relação ao tempo.

$$
-\frac{d C_{A}}{d t}=k C_{A}^{n}
$$

em que $C_{A}$ representa a concentração de antocianinas $\left(\mathrm{mg} \mathrm{L}^{-1}\right)$ no tempo $t, k$ é a constante cinética de degradação do composto e $n$ representa a ordem da reação 0,1 ou 2 .

\subsection{Método Diferencial}

Supôs-se uma expressão de taxa hipotética, Equação 1, a fim de determinar experimentalmente a ordem da reação, $n$, e o valor da constante cinética, $k$, aplicou-se o logaritmo nos dois lados da Equação 1 obtendo-a na forma linearizada, Equação 2.

$$
\ln \left(-\frac{d C_{A}}{d t}\right)=\ln k+n \ln C_{A}
$$

em que $C_{A}$ representa a concentração de antocianinas $\left(\mathrm{mg} \mathrm{L}^{-1}\right)$ no tempo $t$, o coeficiente angular $n$ representa a ordem da reação e o coeficiente linear $k$ é a constante cinética de degradação do composto.

\subsection{Método não-linear}


Integrando a Equação 1 e isolando o termo $t$ obtém se a Equação 3, que posteriormente foi utilizada em conjunto a ferramenta solver no software Excel a fim de obter os parâmetros $k$ e $n$ que minimizem a soma dos quadrados das diferenças entre os valores medidos e os valores calculados para todos os pontos experimentais, Equação 4:

$$
\begin{aligned}
& t_{\text {calc }}=\frac{C_{A \exp }{ }^{1-n}-C_{A 0}{ }^{1-n}}{(n-1) k} \\
& \left(t_{\text {exp }}-t_{\text {calc }}\right)^{2}
\end{aligned}
$$

em que $C_{A e x p}$ é a concentração de antocianinas obtida experimentalmente e $C_{A 0}$ é a concentração inicial de antocianinas, a variável $n$ representa a ordem da reação, $k$ é a constante cinética de degradação do composto, $t_{\exp }$ é o tempo em que as amostras de antocianinas foram coletadas no experimento e $t_{\text {calc }}$ é o tempo obtido pela Equação 3.

Obtenção dos parâmetros cinéticos: a constante cinética $k$ citada nas equações anteriores é dependente da temperatura de acordo com a equação de Arrhenius, Equação (5):

$$
k=k_{0} e^{E a / R T}
$$

em que $k_{0}$ é o fator pré-exponencial $\left(\mathrm{min}^{-1}\right), E a$ é a energia de ativação $\left(\mathrm{J} \mathrm{mol}^{-1}\right)$ e $R$ a constante dos gases $\left(8,314 \mathrm{~J} \mathrm{~mol}^{-1} \mathrm{~K}^{-1}\right)$.

Os parâmetros cinéticos foram determinados utilizando regressão linear dos dados experimentais expressos em logaritmos naturais.

\section{RESULTADOS E DISCUSSÃO}

Para o método integral, o melhor modelo cinético foi o de primeira ordem, o qual obteve o melhor ajuste para os dados experimentais conforme ilustrado na Figura 1.

A Figura 1 ilustra o método integral, utilizando modelo cinético de primeira ordem para o logaritmo natural de $C_{A} / C_{A}$ em função do tempo expresso em minutos para os dados experimentais. Os coeficientes de correlação $\left(\mathrm{R}^{2}\right)$ aproximadamente 0,99 confirmam o bom ajuste dos dados ao modelo.

As constantes de taxa $k$ para as diferentes temperaturas, $60,70,80 \mathrm{e} 90^{\circ} \mathrm{C}$ são obtidas a partir da Figura 1, visto que elas são os coeficientes angulares das equações de reta.

Observa-se que a taxa de degradação de antocianinas $k$ presente na polpa de amora aumenta à medida que a temperatura aumenta, confirmando que quanto maior for a temperatura de operação, maior é a degradação das antocianinas. 
Figura 1- logaritmo natural de $\mathrm{C}_{\mathrm{A} 0} / \mathrm{C}_{\mathrm{A}}$ em função do tempo de reação.

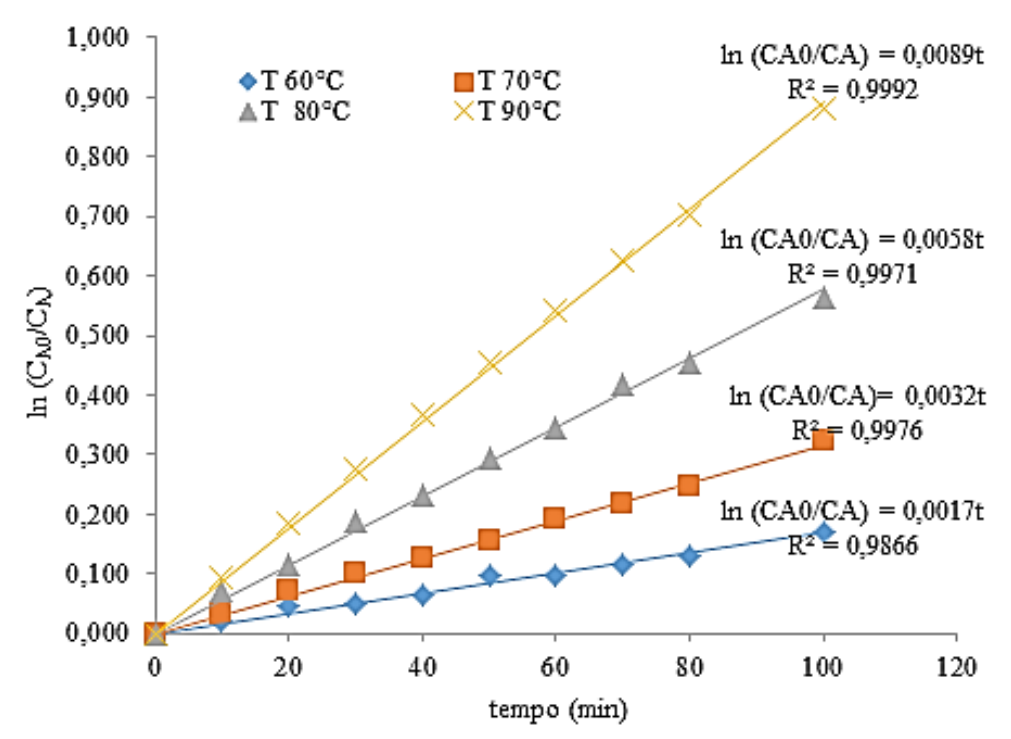

Com os valores das constantes de taxa $k$ para as temperaturas analisadas, os parâmetros cinéticos $k_{o}$ e $E a$ da equação de Arrhenius foram determinados utilizando regressão linear dos dados experimentais expressos em logaritmos naturais como mostra a Figura 2.

Figura 2 - Dependência das constantes cinéticas em relação à temperatura.

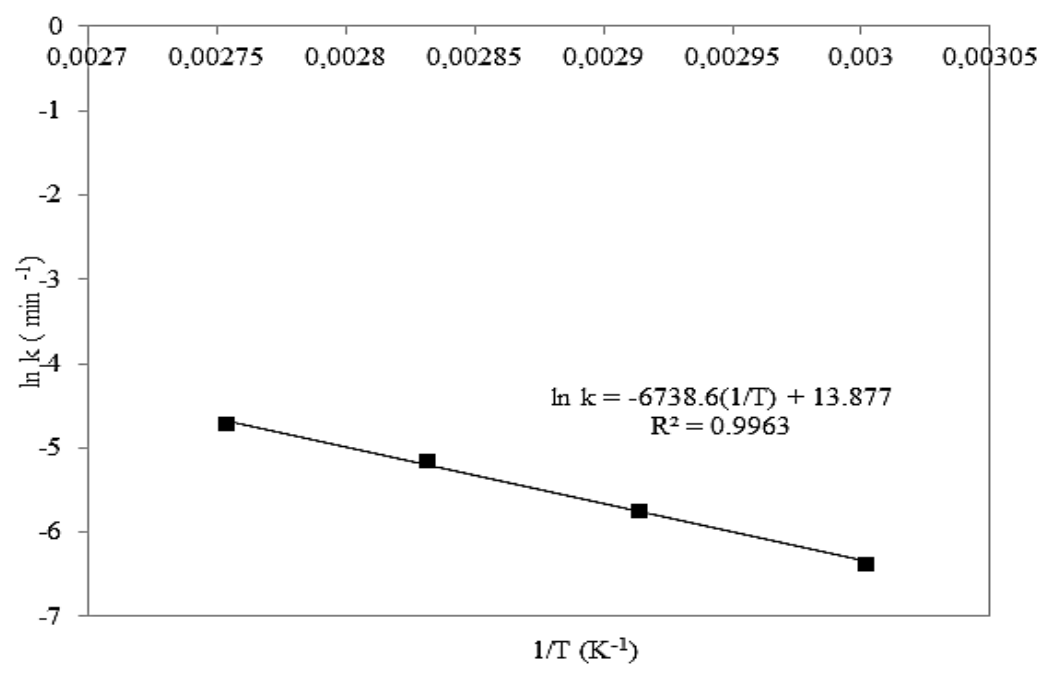

A equação da reta ilustrada na Figura 2 é a forma linearizada da Equação 5, com o fator pré- exponencial $k_{o}$ igual a $1,06 \times 10^{6} \mathrm{~min}^{-1}$ e a energia de ativação $E a$ igual a $56,025 \mathrm{~kJ}$ $\mathrm{mol}^{-1}$. 
$\mathrm{O}$ método de regressão não linear também obteve um modelo cinético de primeira ordem. As constantes de taxa $k$ para as diferentes temperaturas, 60, 70, 80 e $90{ }^{\circ} \mathrm{C}$ foram obtidas através da ferramenta solver assim com a ordem da reação, no qual os valores das constantes também aumentaram com o aumento da temperatura, e com esses valores os parâmetros cinéticos $k_{o}$ e $E a$ da equação de Arrhenius foram determinados utilizando regressão linear dos dados experimentais expressos em logaritmos naturais como mostra a Figura 3.

Figura 3 - Dependência das constantes cinéticas em relação à temperatura.

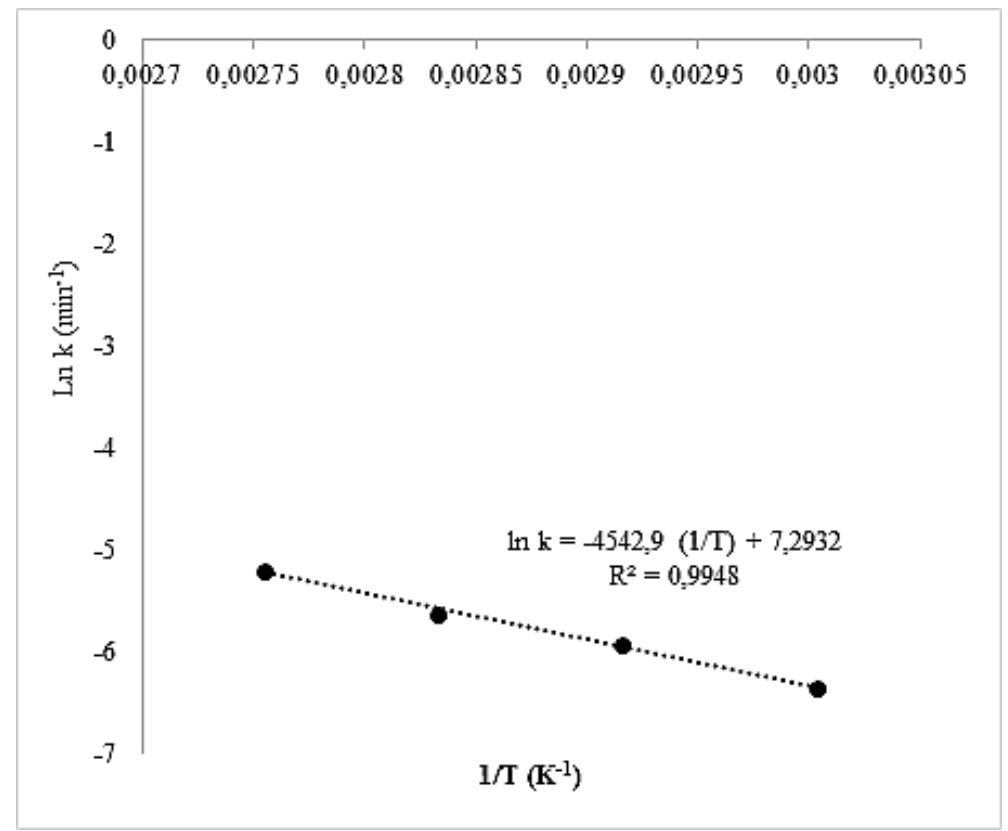

A equação da reta ilustrada pela Figura 3 é a forma linearizada da Equação 5, com o fator pré-exponencial $k_{o}$ igual a $1,470 \times 10^{3} \mathrm{~min}^{-1}$ e a energia de ativação $E a$ igual a $37,770 \mathrm{~kJ}$ $\mathrm{mol}^{-1}$.

Uma análise detalhada das Figuras 2 e 3 sugere uma enorme diferença dos parâmetros da lei de Arrhenius determinados pelo método integral e por regressão não linear. Os parâmetros foram considerados corretos aqueles determinados pelo método integral, visto que o outro pode ter convergido para regiões de mínimos locais, fornecendo, portanto, parâmetros diferentes dos valores reais.

Além disso, o método mais utilizado na literatura é o método integral, em que, para esta pesquisa, o valor da energia de ativação $E a=56,025 \mathrm{~kJ} \mathrm{~mol}^{-1}$ foi bem próximo ao valor relatado por Wang e Xu (2007) na mesma faixa de temperatura, de $60^{\circ} \mathrm{C}$ a $90^{\circ} \mathrm{C}$, para a degradação térmica de antocianinas em amora $\left(E a=58,95 \mathrm{~kJ} \mathrm{~mol}^{-1}\right)$.

A estimativa através do método diferencial não forneceu resultados satisfatórios, pois as equações de reta forneceram coeficientes de correlação linear $\left(\mathrm{R}^{2}\right)$ todos abaixo de 0,9 . Este resultado pode estar associado ao fato do método em questão fornecer uma aproximação para a derivada da equação de taxa, ou seja, valores médios. Esses valores médios podem ser 
bastante imprecisos em virtude de os pontos experimentais terem sido coletados em intervalos de tempo não muito próximos.

\section{CONCLUSÃO}

O objetivo deste trabalho foi avaliar os métodos de integral, diferencial e não linear para obter um modelo cinético com parâmetros válidos para operar. Os métodos integral e a regressão não linear foram os métodos que obtiveram os melhores resultados. Ambos forneceram um modelo cinético de primeira ordem para a taxa de degradação térmica de antocianinas, fornecendo parâmetros de acordo com pesquisas disponíveis na literatura.

\section{REFERÊNCIAS}

ALKEMA, S.; SEAGER, S.L. The Chemical Pigments of Plants; Journal of Chemical Education. Ogden (Utah), mar, 1982.

LEE, J., DURST, R.W., WROLSTAD, R.E., Determination of Total Monomeric Anthocyanin Pigment Content of Fruit Juices, Beverages, Natural Colorants, and Wines by the pH Differential Method: Collaborative Study. J. AOAC Int. 88, 1269-1278, 2005.

LOPES. T.J; XAVIER. M. F; QUADRI. M. G. N; QUADRI. B. Q., Antocianinas: uma breve revisão das características estruturais e da estabilidade. Agrociência, Pelotas, v.13, n.3, p. 291-297, jul-set, 2007.

KUMAR, S.; PANDEY, A. K., Chemistry and Biological Activities of Flavonoids: An Overview. The Scientificworld Journal. Allahabad, out, 2013.

KUSKOSKI, E. M.; ASUERO, A. G.; MORALES, M. T.; FETT, R., Frutos tropicais silvestres e polpas de frutas congeladas: atividade antioxidante, polifenóis $e$ antocianinas. Ciência Rural, Santa Maria, v.36, n.4, p.1283-1287, jul-ago, 2006.

WANG, W-D.; XU, S-Y., Degradation kinetics of anthocyanins in blackberry juice and concentrate. Journal of Food Engineering, out, 2007. 